\title{
Reflexões sobre o Serviço de Medicina Interna do Hospital de Guimarães
}

\section{Reflections on The Internal Medicine Service of Hospital De Guimarães}

\section{Jorge Cotter}

Palavras-chave: Departamentos Hospitalares; Medicina Interna.

Keywords: Hospital Departments; Internal Medicine.

O Serviço de Medicina do Hospital de Guimarães existe pelo menos desde o século XVI. Inicialmente a preponderância dos doentes era a de Medicina "Externa", quadros clínicos relacionados com a Venereologia, a qual, nesse tempo, ocupava um piso dos dois existentes no Hospital. Em 1843, o Hospital instala-se no antigo Convento dos Capuchos, junto ao Castelo de Guimarães. Durante muitos anos existiram dois Serviços de Medicina, que foram fundidos com a criação do Serviço Nacional de Saúde. Nos anos 80 do século passado, o Serviço de Medicina Interna estava em modernas instalações, com uma lotação de 43 camas e sob a direcção do Dr. Artur Vieira e Brito, que chefiou o Serviço cerca de 30 anos. Em 1989, ainda no Hospital de Santo António dos Capuchos, - Serviço solicita e é-Ihe atribuída a idoneidade total para a formação de internos de medicina interna. À data, o quadro médico era composto por dois Chefes de Serviço e cinco Assistentes Hospitalares. Em Setembro de 1991, o Hospital de Guimarães passa para as novas instalações, num edifício construído de raiz. O Serviço de Medicina Interna passa a dispor de 90 camas de internamento e um quadro médico de 20 médicos, entre assistentes Hospitalares e Chefes de Serviço. Desde então, o Serviço tem-se expandido progressivamente, dispondo na presente data de um quadro de 22 Assistentes Hospitalares, 6 Assistentes Hospitalares Graduados e 3 Assistentes Hospitalares Graduados Seniores. Tem 28 Internos Complementares de Medicina Interna em formação.

Como em todos os Serviços de Medicina Interna, o Serviço no Hospital de Guimarães privilegia o Método Clínico como forma de abordagem dos pacientes que para si são orientados. Costumamos dizer que o âmbito das patologias é o descrito nos grandes tratados de medicina interna. Há, no entanto, algumas excepções, das quais são exemplos os doentes sob ventilação invasiva (internados nos cuidados

Diretor Serviço de Medicina Interna, Hospital Senhora da Oliveira Guimarães, Portugal

DOI: 10.24950/Opiniao/Jorge Cotter/H.Guimaraes/4/2020 intensivos) e os pacientes com cardiopatia isquémica aguda (internados em unidade coronária). Na prática, o Serviço tenta ir mais além, através da execução de um conjunto de procedimentos que alargam a sua autonomia e permitem uma maior celeridade nas pesquisas diagnósticas e práticas terapêuticas. Nestas situações, estamos a falar da realização de exames, por Internistas e Internos de Formação Específica de Medicina Interna, dos quais são exemplo, a prática de biópsias hepáticas, renais, ósseas e ganglionares e do ponto de vista terapêutico, a drenagem de pneumotóraces, empiemas e abcessos hepáticos, muitas das situações com controlo ecográfico em parte já realizado pelos Internistas do Serviço.

A actividade do Serviço expande-se por diversas áreas, nomeadamente assistencial, pedagógica e de investigação clínica.

No campo assistencial, o Serviço actua no Internamento, Serviço de Urgência, Consultadoria Interna, Consulta Externa, Hospitalização Domiciliária e Unidade Médica Intermédia/Unidade de Acidentes Agudos Cerebrovasculares (AVCs).

No Internamento, dispõe na presente data, de 101 camas, com três alas de 29 camas e uma ala de 14 camas. Tem 8 camas de pressão negativa e está em vias de dispor de 20 camas, as quais aumentam muito a segurança no tratamento dos pacientes com tuberculose activa e daqueles com COVID-19. Realiza-se uma visita conjunta semanal a cada ala do Serviço (presentemente adaptada à conjuntura COVID-19), com a presença do Director de Serviço, com uma duração média de 90 minutos. Os Internos de Formação Específica são ensinados a apresentar um doente em cerca de três minutos. Os casos mais complexos, são objecto de análise conjunta mais aprofundada em qualquer dia, sem horário definido. O número de internamentos tem-se mantido estável nos últimos anos (Fig. 1), com 5774 no ano de 2019, com um índice de case-mix de 1,25, um tempo médio de internamento clínico de 7,8 dias e uma taxa de mortalidade de 13,1\%.

A Consulta Externa encontra-se subdividida em várias áreas: existe uma consulta de Medicina Interna Global e um conjunto de consultas temáticas que obedecem a múltiplos protocolos de actuação. As consultas temáticas são as de Medicina Interna/Doenças Autoimunes, Medicina Interna/ Doenças Infecciosas, Medicina Interna/Diabetologia e Medicina Interna/Hipertensão e Risco Cardiovascular e Doenças Renais. As duas últimas dão o suporte clínico ao Centro de 


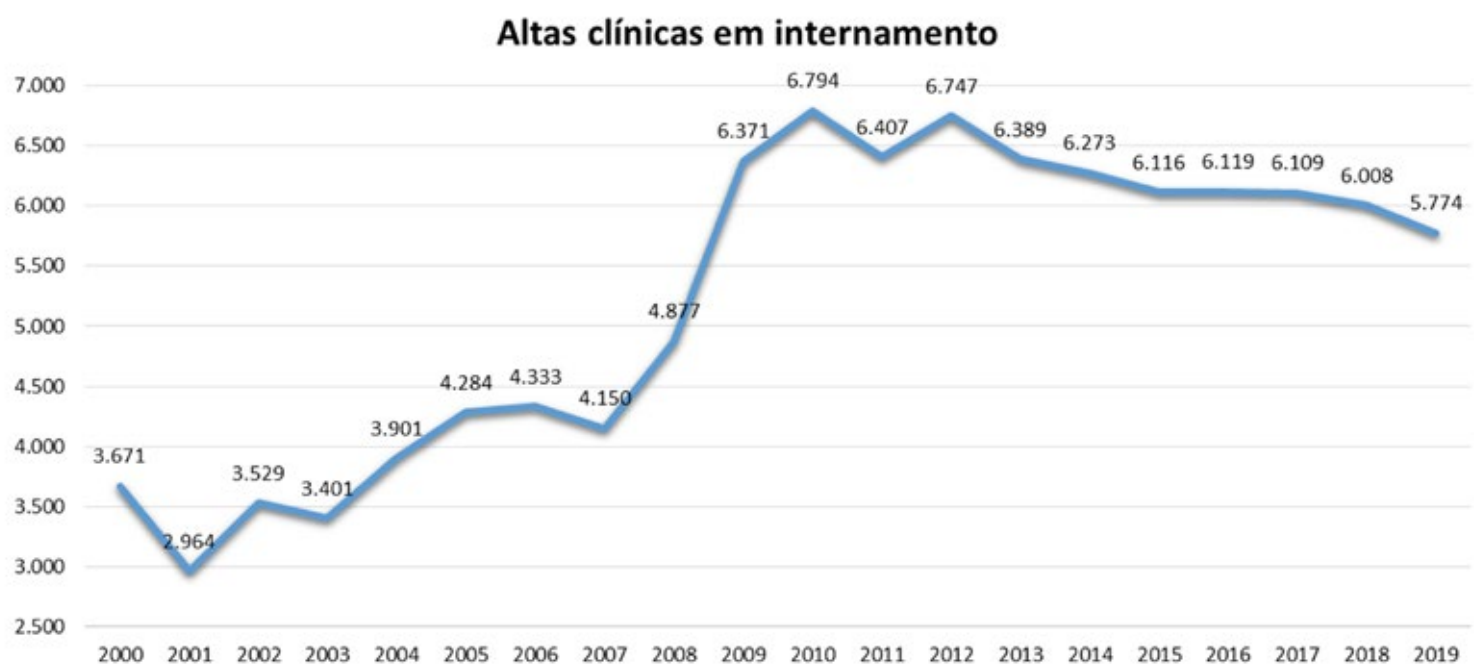

Figura 1: Altas clínicas em internamento

Investigação e Tratamento em Hipertensão e Risco Cardiovascular Global, do qual o Serviço é Centro de Referência Europeu. Em termos globais, o Serviço disponibiliza cerca de 350 horas semanais oficiais de consultas, com 23661 consultas realizadas durante o ano de 2019, das quais 3754 foram primeiras consultas (Fig. 2).

A Consultadoria Interna é realizada a todos os Serviços do Hospital com camas de internamento. Obedece a uma distribuição por escala, onde estão emparelhados um Assistente Hospitalar e um Interno Complementar acima do $1^{\circ}$ Ano. Há o objectivo de responder a todas as solicitações no prazo máximo de 24 horas, nos dias úteis.

O Serviço de Urgência é assegurado presencialmente todos os dias durante as 24 horas. Em condições normais, cada Equipa de Medicina Interna tem dois Assistentes Hospitalares que asseguram 24 horas de Urgência a cada semana e dois Internos Complementares que asseguram 12 horas de Urgência por semana. Em período de férias, podem os Internos Complementares efectuar um período de mais 12 horas por semana, nunca seguidos em relação ao período normal. No Serviço de Urgência, os médicos de Medicina Interna respondem pela Sala de Emergência, pelos doentes triados pelo protocolo Manchester com a cor laranja e pela Sala de Observações. Os restantes pacientes têm de ser previamente triados e se solicitado, podem ser ulteriormente analisados.

A Hospitalização Domiciliária é também assegurada

Número de consultas

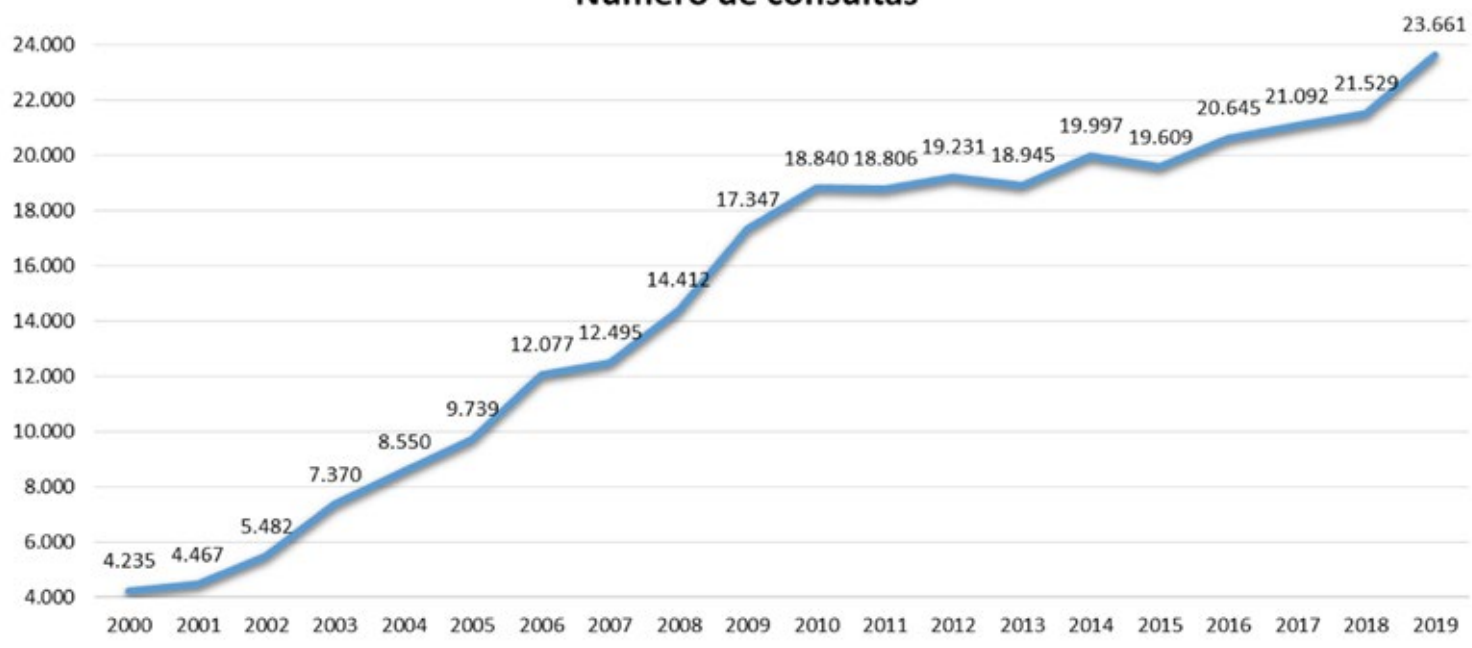

Figura 2: Número de consultas 
pelo Serviço de Medicina Interna. Obedece a um esquema de escala rotativa mensal, que engloba um Assistente Hospitalar não graduado e um Interno Complementar do último ano. Pareceu-nos redutor para o trabalho de um Internista ter um quadro estanque nesta área e esta decisão prática foi consensual no Serviço. Embora tenhamos sérias dúvidas no momento actual sobre a relação custo/benefício da hospitalização domiciliária, entendemos a prioridade política e respondemos ao que nos é solicitado.

O Serviço de Medicina Interna dispõe, dentro do seu espaço físico, de uma Unidade Intermédia Médica e uma Unidade de AVCs, com 12 camas, sem limitação estanque entre as duas. Demorou 12 anos a ser satisfeita esta reivindicação que entendíamos essencial para o tratamento dos doentes mais graves e instáveis. Está aberta desde 01/01/2020 e funciona com um quadro médico e de enfermagem específico. Fora do horário de rotina, a presença médica é assegurada presencialmente por um Assistente Hospitalar e por um Interno Complementar que nas actuais circunstâncias de escassez de recursos médicos, funcionam também como residentes do Serviço.

Do ponto de vista pedagógico, o Serviço dá apoio prático aos $3^{\circ}, 4^{\circ}, 5^{\circ}$ e $6^{\circ}$ anos do Curso de Medicina da Universidade do Minho, desde 2003, altura em que os alunos do $1^{\circ}$ curso chegaram ao ciclo clínico. Habitualmente recebe 12 turmas com 4-5 estudantes cada e no final de cada ciclo, efectuam-se exames práticos com doentes, nos $4^{\circ}$ e $5^{\circ}$ anos.

Na área da Investigação Clínica e Pós-Graduação, o Serviço de Medicina Interna é, desde 2010, Centro de Referência Europeu em Hipertensão e Risco Cardiovascular Global, atribuição dada pela European Society of Hypertension and Cardiovascular Risk. Correspondeu à satisfação de um conjunto de condições do ponto de vista assistencial clínico e de publicações científicas em revistas indexadas de reconhecido mérito. Gerou duas teses de doutoramento, está a orientar ou co-orientar mais três teses e ainda sete teses de mestrado. Foi escolhido para estágios de formação e/ou investigação por médicos de diferentes Instituições Universitárias, Países e Continentes [(Portugal (2), Angola (3), Brasil (5), México (1), Espanha (6) e Suécia (1)].

O Serviço criou condições para que um dos seus membros assumisse as seguintes responsabilidades em instituições científicas internacionais: a) Presidência do Working Group on Hypertension and the Brain da European Society of Hypertension (ESH); b) Vice-Presidência do Working Group on Vascular Structure and Function, da ESH; c) Comité Executivo da Artery Society; d) Fundação e Coordenação desde Novembro de 2017, da Rede Ibérica de Investigação em Estrutura Arterial, Hemodinâmica Central e Neurocognição, envolvendo 18 Centros Clínicos e Académicos da Península Ibérica dedicados à investigação destes temas; e) Membro dos conselhos editoriais de revistas indexadas e com peer-review (Current Hypertension Reviews e International Journal of Cardiology and Hypertension); f) Revisor convidado de diferentes revistas nacionais e internacionais: Journal of Hypertension, European Journal of Clinical Investigation, Mayo Clinical Proceedings, Journal of Human Hypertension, International Journal of Cardiology and Hypertension, International Journal of Medical Informatics, Annals of Internal Medicine, Revista Portuguesa de Medicina Interna e Artery Research.

Até ao momento, o Serviço produziu 49 publicações em revistas indexadas e com peer-review, para além de centenas de comunicações científicas e centenas de conferências em reuniões nacionais e internacionais.

Naturalmente que a gestão de um Serviço de Medicina Interna com a dimensão descrita não se afigura fácil. Para além do cerne da questão que é a do seu âmbito, ou seja, avaliar, diagnosticar e tratar todos os pacientes com as patologias descritas nos grandes Tratados de Medicina Interna, a descentralização em vários níveis de decisão afigura-se-nos fundamental. Se do ponto de vista assistencial ninguém nega o contributo que pode e deve ser dado pelas diversas subespecialidades médicas e serviços cirúrgicos, a última decisão na orientação diagnóstica e de tratamento deve ser do Internista, logo, do Serviço. Dentro do Serviço, sempre que possível, dada a complexidade do exercício da medicina actual, as decisões devem ser colegiais, muitas vezes apoiadas em algoritmos de decisão clínica, mas sempre adequadas ao caso concreto em questão. Pode-se assim dizer que as decisões clínicas são do Serviço e não de qualquer médico individual.

No Serviço de Medicina Interna do Hospital de Guimarães, no que toca à gestão corrente, embora a última decisão caiba ao Director de Serviço, existe uma ampla descentralização de decisões, dependendo dos assuntos e da sua complexidade. Cada Chefe de Ala do Internamento, correspondente a cerca de 30 camas e cada responsável pela gestão de cada Consulta Temática tem grande autonomia nas decisões que ao seu pelouro digam respeito. O mesmo se passa na Hospitalização Domiciliária, no Centro de Investigação e na Unidade Intermédia Médica e de AVCs, onde os seus responsáveis têm uma vasta capacidade decisória. Todos os elementos do Serviço podem ser ouvidos, dependendo dos assuntos, complexidade e interesse dessas opiniões para o Serviço. Cabe ao Director de Serviço fazer a síntese e com a sua experiência, ajudar a moldar as posições que o Serviço possa manifestar em termos filosóficos ou de prática médica. Um exemplo prático e actual diz respeito à posição do Serviço quanto aos seus Internos de Formação Específica; os Internos são do Serviço e como tal cabe ao Serviço assegurar igualdade de oportunidades na aprendizagem teórica, prática assistencial e na investigação; daí, todos trabalharem com todos, no Serviço, ao longo do Internato. No final de cada ano, a avaliação individual é discutida em reunião, com todos os assistentes hospitalares; as notas atribuídas são assim fruto de um consenso a nível global do Serviço de Medicina Interna.

Qualquer problema de trabalho que surja com um 
elemento do Serviço, desde que complexo e com interesse para os outros membros do Serviço, pode, deve e é discutido colegialmente para permitir actuações concertadas. Isso tem permitido uma posição robusta quando estão em causa princípios ou valores dos quais os Internistas não podem abrir mão.

Um Serviço de Medicina Interna uno no essencial e diverso na heterogeneidade pessoal, de conhecimento e de interesses nas diversas áreas da medicina, é primordial para a Medicina Interna continuar a ser o pilar fundamental do Sistema de Saúde; para isso, é necessário promover o diálogo, a responsabilização consciente e a noção de que uma vez discutidos colegialmente os problemas, a posição do Serviço tem que ser assumida por todos.

O Serviço de Medicina Interna do Hospital Senhora da Oliveira em Guimarães tem assim, ao longo do tempo, efectuado as suas adaptações, de molde a promover uma Medicina Interna cada vez mais interventiva e envolvente, a bem dos doentes e do Hospital em geral.

\section{Responsabilidades Éticas}

Conflitos de Interesse: Os autores declaram a inexistência de conflitos de interesse na realização do presente trabalho.

Fontes de Financiamento: Não existiram fontes externas de financiamento para a realização deste artigo.
Proveniência e Revisão por Pares: Não comissionado; revisão externa por pares.

\section{Ethical Disclosures}

Conflicts of interest: The authors have no conflicts of interest to declare. Financing Support: This work has not received any contribution, grant or scholarship

Provenance and Peer Review: Not commissioned; externally peer reviewed.

(c) Autor (es) (ou seu (s) empregador (es)) e Revista SPMI 2020. Reutilização permitida de acordo com CC BY-NC. Nenhuma reutilização comercial. (c) Author(s) (or their employer(s)) and SPMI Journal 2020. Re-use permitted under CC BY-NC. No commercial re-use.

\section{Correspondence / Correspondência:}

Jorge Cotter - medicina@hospitaldeguimaraes.min-saude.pt

Diretor Serviço de Medicina Interna, Hospital Senhora da Oliveira, Guimarães, Portugal

Rua dos Cutileiros, Creixomil, 4835-044 Guimarães

Received / Recebido: 07/10/2020

Accepted / Aceite: 07/10/2020

Publicado / Published: 18 de Dezembro de 2020 